\title{
Interatomic potentials for elastically isotropic crystals
}

\author{
Reza Najafabadi and David J Srolovitz \\ Department of Materials Science and Engineering, University of Michigan, Ann Arbor, MI \\ 48109, USA
}

Received 3 December 1994, accepted for publication 30 May 1995

\begin{abstract}
We introduce a simple approach to fitting interatomic potentials to represent elastically isotropic solids, in order to make direct comparisons with the predictions of isotropic linear elasticity. The potentials are of the Finnis-Sinclair type and are parametrized to fit Au and Ag. Under uniaxial loading, it was shown that the solids modelled employing the 'elastically isotropic' potentials remain elastically isotropic for strains of $0.5 \%$ or less. The properties of the (001), (011) and (111) surfaces were determined and compared with those of elastically anisotropic $\mathrm{Au}$ and $\mathrm{Ag}$. It was also found that the surface energy anisotropy is lower in solids modelled employing 'elastically isotropic' potentials than in those represented by elastically anısotropic potentials.
\end{abstract}

\section{Introduction}

Atomistic simulations provide an excellent complement to elastic theories in describing the structure of defects in crystals. Isotropic linear elasticity has been very successful in describing atomic displacements far from the defect but is inapplicable near the defect where the small strain assumption of linear elasticity is invalid. It is in describing the structure of the core region that atomistic simulations of defects have proven invaluable. In order to understand the detailed properties of defects using both atomistic and elasticity approaches [1], it is imperative that the two approaches are consistent. For example, the elastic constants used in the elastic calculations must be consistent with the interatomic potentials used in the atomistic study. Making sure that the elastic and atomistic methods are consistent with each other is often very difficult owing to the inherent complexity of solving for the anisotropic elastic fields of defects, such as dislocations, grain boundaries, steps on surfaces, and point defects. Because of these difficulties associated with obtaining anisotropic solutions, it would greatly simplify these studies if isotropic, linear elasticity could be used instead of anisotropic elasticity. In order to do this, however, we must ensure that the interatomic potentials employed in the atomistic simulations yields isotropic linear elastic results in the small strain limit. This paper addresses the development of interatomic potentials which are consistent with isotropic linear elasticity.

The structures of the cores of defects in crystals are often difficult to analyse because there are often competing effects that synergistically determine the structure. Therefore, one of the prime benefits of using elastically isotropic interatomic potentials is to allow one to separate the effects of anisotropy from other effects, such as anharmonicity. Using these interatomic potentials, we have been able to show unambiguously that the excess volume (expansion) associated with twist grain boundaries is attributable largely to the anharmonicity of the atomic interactions and only weakly to the elastic anisotropy [2]. Further, in cases 
where a detailed elastic model of the defect is not known, it is advantageous to investigate several competing models. While this may, in principle, be done using anisotropic elasticity, the simplifications inherent to isotropic elasticity greatly simplify the analysis and therefore makes it easier to investigate many models. For example, isotropic elastic models of steps or adatoms on surfaces are much simpler than the corresponding anisotropic models.

We present a set of many-body interatomic potentials of the Finnis-Sinclair (FS) [3] type which describe elastically isotropic, cubic crystals. In order to assess the effects of modifying the original interatomic potentials that describe anisotropic solids, we present several comparisons of surface data obtained using the isotropic and anisotropic elastic potentials.

\section{Interatomic potentials}

The basic interatomic potentials which we analyse and modify here are of the many-body FS [3] type. In both the FS and the embedded atom method (EAM) [4] potentials, the energy of an atom is described in terms of a central pair potential $V_{i j}$ and an embedding function $f$ as

$$
U_{i}=\frac{1}{2} \sum_{j} V_{i j}-f\left(\rho_{i}\right)
$$

where

$$
\rho_{i}=\sum_{j} \phi_{i j}
$$

and both $V_{i j}$ and $\phi_{i j}$ are functions of only the interatomic distance $r_{i j}$. In the FS model the embedding function is given simply as $f(\rho)=\sqrt{\rho}$.

To determine the functions $V_{i j}$ and $\phi_{i j}$ for the FCC metals considered here, we follow the procedure developed by Ackland et al [5] and express these functions as

$$
\begin{aligned}
& V(r)=\sum_{k=1}^{6} a_{k}\left(r_{k}-r\right)^{3} H\left(r_{k}-r\right) \\
& \phi(r)=\sum_{k=1}^{2} A_{k}\left(R_{k}-r\right)^{3} H\left(R_{k}-r\right)
\end{aligned}
$$

where $H$ is a Heaviside function $(H(x)=0$ for $x<0$ and $H(x)=1$ for $x>1$ ). As in [5], the knot points $r_{k}$ and $R_{k}$ are first chosen and then the sublimation energy, lattice constant, elastic constants, vacancy formation energy and stacking-fault energy of the metal of interest are employed to determine the fitting parameters $a_{k}$ and $A_{k}$. As was noted in [5] for $r_{6}<r_{n n}\left(r_{n n}\right.$ is the nearest-neighbour distance), the above equilibrium properties do not depend upon $a_{6}$ and hence this parameter may be determined from the properties of the metal at higher densities (lattice constants smaller than the zero-pressure lattice constant $a_{0}$ ). We choose $a_{6}$ to yield the best fit between our calculated energies and those determined using the universal binding energy relation [6] as a function of lattice constant.

Cubic crystals have three independent elastic constants. This number is reduced to two in an isotropic elastic solid, through the following equation:

$$
C_{11}-C_{12}=2 C_{44}
$$

where the above elastic constants are defined in the principal-axes coordinates.

In order to develop interatomic potentials to describe $\mathrm{Ag}$ and $\mathrm{Au}$ as isotropic elastic solids, we employed the equilibrium properties of $\mathrm{Ag}$ and $\mathrm{Au}$ as given in table 1 to determine 
Table 1. Properties of Au and Ag used to fit the potentials. $E_{s u b}$ is the sublimation energy, $a_{0}$ is the lattice parameter, $E_{v}$ is the vacancy formation energy, $E_{s f}$ is the stacking-fault energy, and the $C_{i j}$-values are elastic constants. The modified $C_{11}$ and $C_{12}$ used in the development of 'elastically isotropic' potentials I and II are also tisted.

\begin{tabular}{lllllll}
\hline & Au & Au I & Au II & Ag & Ag I & Ag II \\
\hline$E_{s u b}(\mathrm{eV})$ & 3.787 & 3.787 & 3.787 & 2.967 & 2.967 & 2.967 \\
$a_{0}(\AA)$ & 4.078 & 4.078 & 4.078 & 4.086 & 4.086 & 4.086 \\
$E_{v}(\mathrm{eV})$ & 0.940 & 0.940 & 0.940 & 1.000 & 1.000 & 1.000 \\
$E_{s f}(\mathrm{eV})$ & 0.007 & 0.007 & 0.007 & 0.007 & 0.007 & 0.007 \\
$C_{\mathrm{l} 1}\left(10^{11} \mathrm{~N} \mathrm{~m}^{-2}\right)$ & 1.860 & 2.410 & 2.227 & 1.240 & 1.856 & 1.651 \\
$C_{\mathrm{l} 2}\left(10^{11} \mathrm{~N} \mathrm{~m}^{-2}\right)$ & 1.570 & 1.570 & 1.387 & 0.934 & 0.934 & 0.729 \\
$C_{44}\left(10^{11} \mathrm{~N} \mathrm{~m}^{-2}\right)$ & 0.420 & 0.420 & 0.420 & 0.461 & 0.461 & 0.461 \\
\hline
\end{tabular}

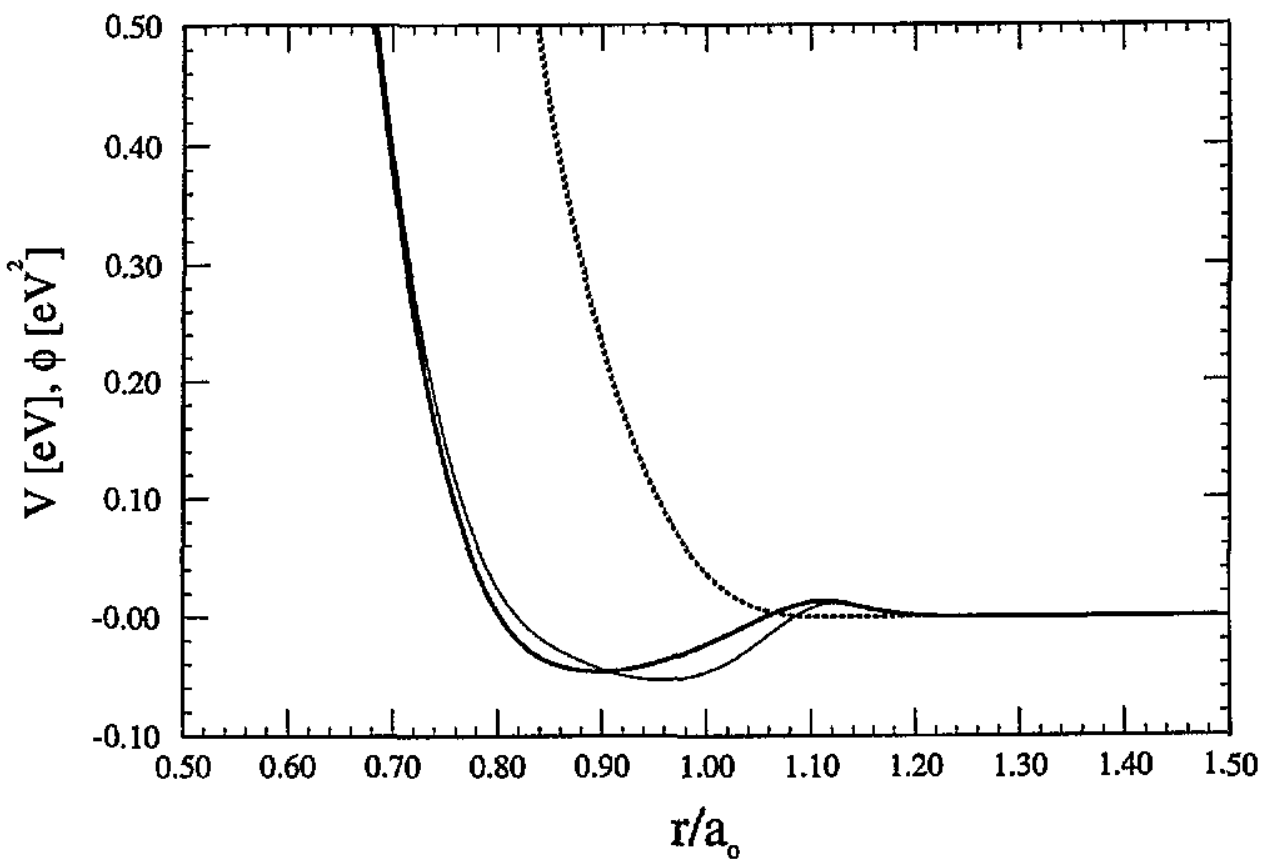

Figure 1. The variation in the potential functions $V$ (solid curve) and $\phi$ (dotted curve) with distance for elastically anisotropic Au (thick curves) and elastically isotropic pseudo-Au I (thin curves).

the fitting parameters $a_{k}$ and $A_{k}$ in equations (2). The elastic constants used in determining the fitting parameters were modified to satisfy the isotropy condition (equation (3)). We choose two simple and distinct ways in which to satisfy this condition. In the first (I), we write the Lamé constants as $\mu=C_{44}$ and $\lambda=C_{12}$, where the $C_{i j}$ are the anisotropic elastic constants of $\mathrm{Ag}$ or $\mathrm{Au}$. In the second approach (II), we guarantee that both the isotropic and the anisotropic solids have the same shear modulus $C_{44}$ and bulk modulus. These modified elastic constants are presented in table 1. The fitting parameters for these two sets of potentials describing elastically isotropic crystals are given in table 2 together with those for the elastically anisotropic potentials. Note that there are slight differences between the 
parameters given in table 2 for Au and those previously reported [5] due to minor changes made in the positions of the knot points in the present study.

Figure 1 shows the variations in $V$ and $\phi$ with the interatomic distance $r$ for both $A u$ and pseudo-Au I (isotropic case I). There are no qualitative differences between the two $V$ functions, and the two $\phi$ functions are essentially indistinguishable (this can also be seen from the values of fitting parameters given in table 2). The $V$ and $\phi$ functions corresponding to the pseudo-Au II (isotropic case II) are similar to those in figure 1 and the corresponding results for $\mathrm{Ag}$ and pseudo-Ag potentials show similar differences to those for Au.

As expected, these 'elastically isotropic' potentials predict that the FCC lattice is more stable than the HCP lattice, consistent with the positive stacking-fault energies employed in the fitting procedure. The relaxed intrinsic and extrinsic stacking-fault energies corresponding to these potentials were determined and all are approximately $13 \pm 0.5 \mathrm{~mJ} \mathrm{~m}^{-2}$. The relative stability of the FCC structure compared with the $\mathrm{BCC}$ structure (employing these 'elastically isotropic' potentials) is indicated in figure 2 where we show the variation in energy with atomic volume for both the FCC and the BCC structures. At zero pressure (where the slope of the energy curve is zero) the energy of the FCC structure is approximately $0.2 \mathrm{eV}$ smaller than that corresponding to the BCC structure. For the anisotropic potentials, the results show similar trends. Figure 2 also shows the energy predicted by the universal binding energy relation [6] which was used in determining the fitting coefficients.

\section{Uniaxial load response}

An elastically isotropic solid responds similarly to loading in different directions. For example, when a solid is uniaxially stressed (i.e. relaxation is allowed in the plane normal to the load direction), the state of strain is independent of the load direction. Figure $3(a)$ shows the result of such an 'experiment' in which we employed the pseudo-Au I potential to calculate the strain $\varepsilon_{z z}$ as a function of the stress $\sigma_{z z}$ where the $z$ direction was chosen to lie along the $\langle 001\},\{011\rangle$ and $\langle 111\rangle$ directions. Figure $3(b)$ shows the results of similar calculations using the 'elastically anisotropic' Au potential. In the stress-free state, the slopes of the different stress-strain curves corresponding to the psuedo-Au I potential (figure $3(a)$ ) are all the same (i.e. Young's modulus is independent of the load direction), while those obtained using the 'elastically anisotropic' Au potential (figure $3(b)$ ) are all different. Similar results were found for the other potentials. We note that, although we determined the stress-strain curves for only three high-symmetry directions, stress-strain curves for all other load axes will lie between these.

Figure $3(b)$ also shows that, beyond approximately $0.5 \%$ strain (compressive and tensile), linear elasticity begins to break down and, therefore, the non-linear elastic terms (higher-order elastic constants) become important. The stress-strain curve corresponding to the pseudo-Au I potential (figure $3(a)$ ) and those corresponding to the other isotropic potentials are also linear within a similar strain range $(0.5 \%)$. We note that the points where the stress-strain curves in figure $3(a)$ and those for the other 'elastically isotropic' potentials start to diverge coincide with the point where they start to deviate from linear functions. This is simply because while the second-order elastic constants corresponding to these potentials satisfy the isotropy condition, the higher-order elastic constants are not isotropic.

For an elastically isotropic solid the Poisson ratio $-\varepsilon_{x x} / \varepsilon_{z z}$ should not vary as the load direction changes. The transverse strain versus normal strain curves for the three load directions are plotted in figure 4 for both the pseudo-Au I and the 'elastically anisotropic' Au potentials. Again, as expected for an isotropic crystal, the slopes of the transverse strain 


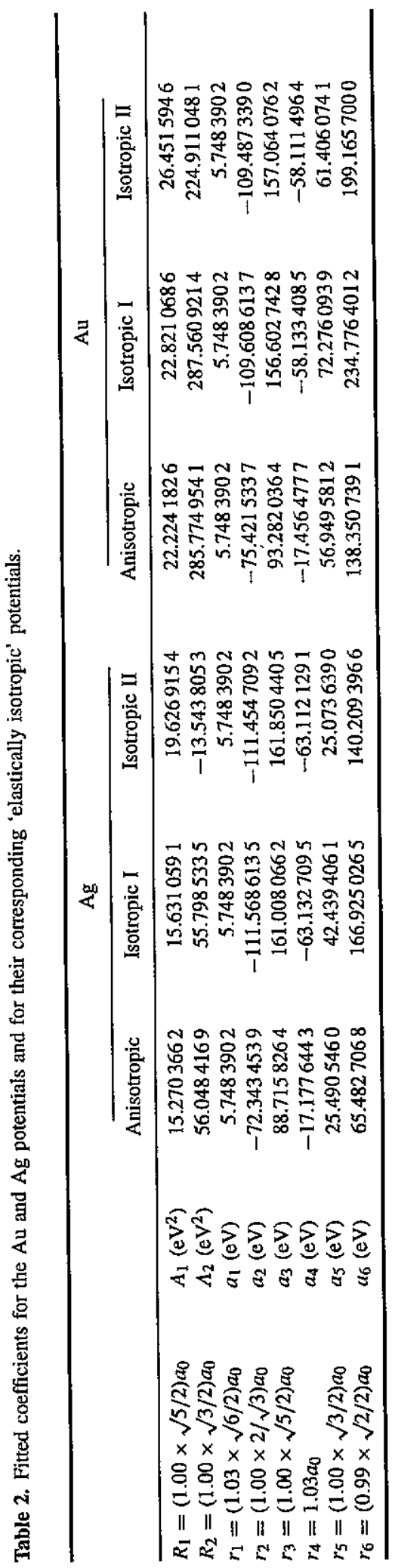



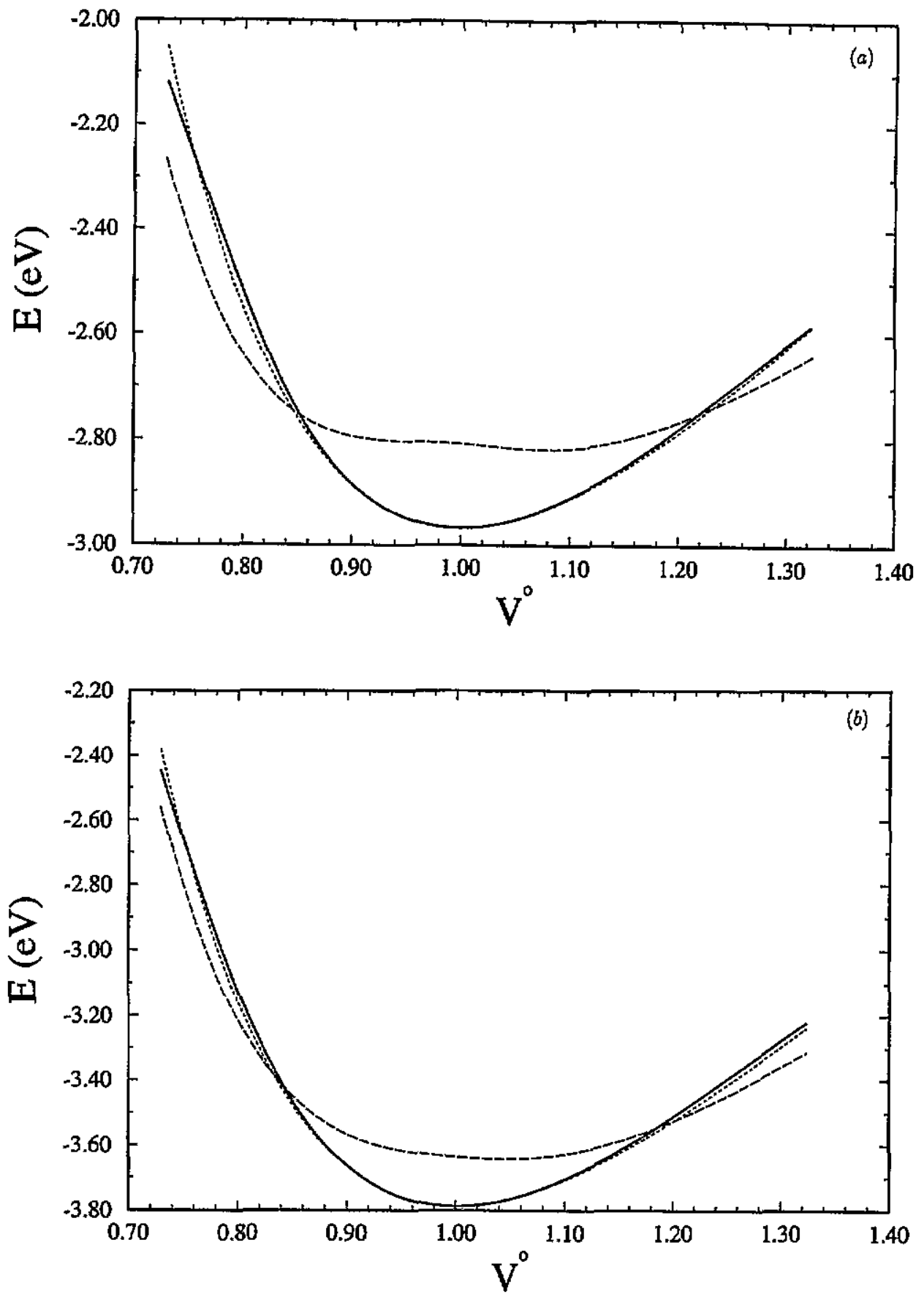

Figure 2. The variation in the energy per atom of an FCC and a BCC crystal model with atomic volume. The energy is determined employing the 'elastically isotropic' (a) Ag II potentials and (b) Au II potentials. The solid and broken curves represent FCC and BCC crystals, respectively. The dotted curve shows the energy determined using the universal binding energy relation [6]. 

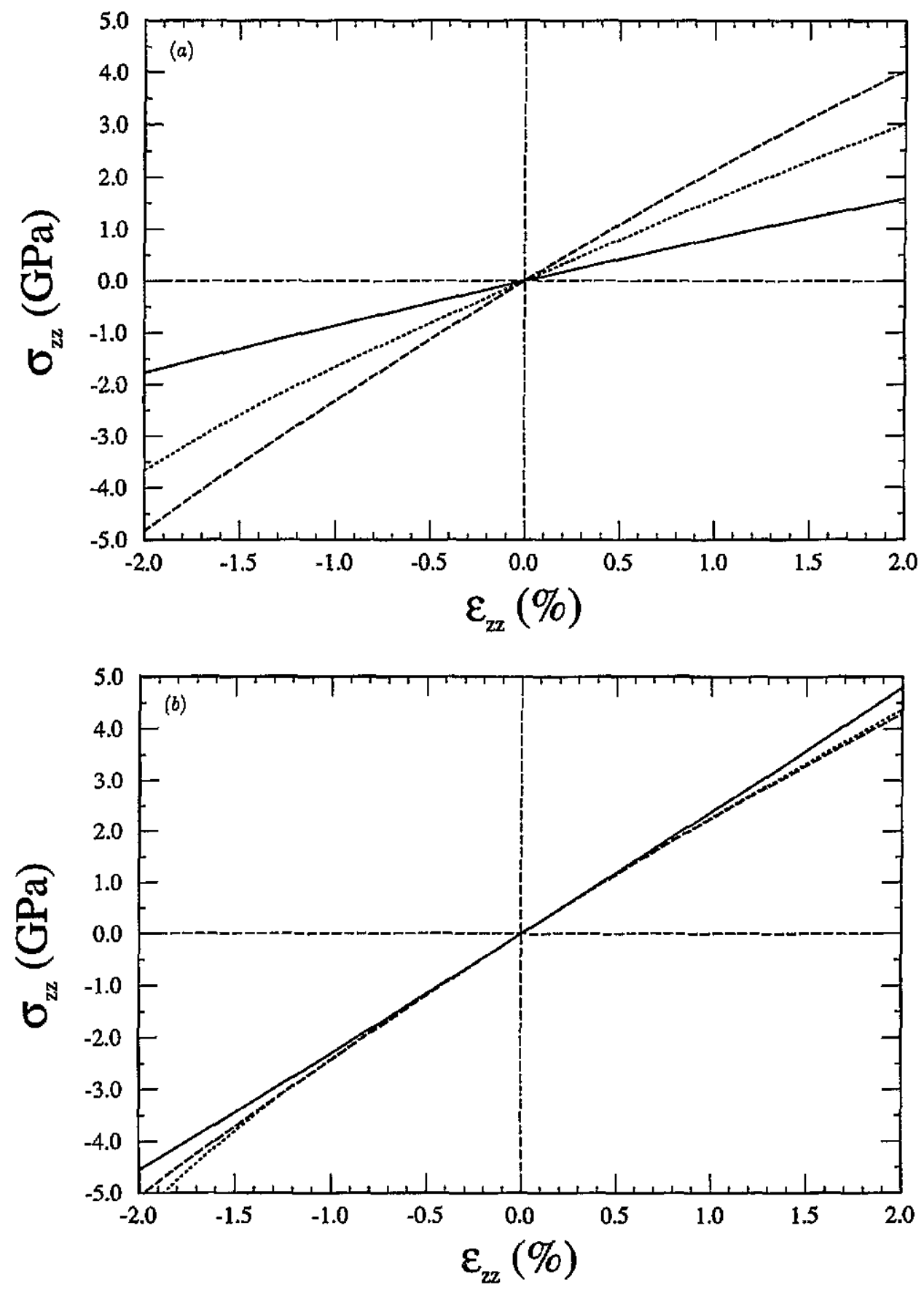

Figure 3. Stress-strain curves for the load direction $\langle 001\rangle$ (solid curve), $\langle 011\rangle$ (dotted curve) and $\langle 111\rangle$ (broken curve) determined employing $(a)$ the Au potential and $(b)$ the 'elastically isotropic' Au I potential. Stresses in the plane normal to the load direction are zero. 

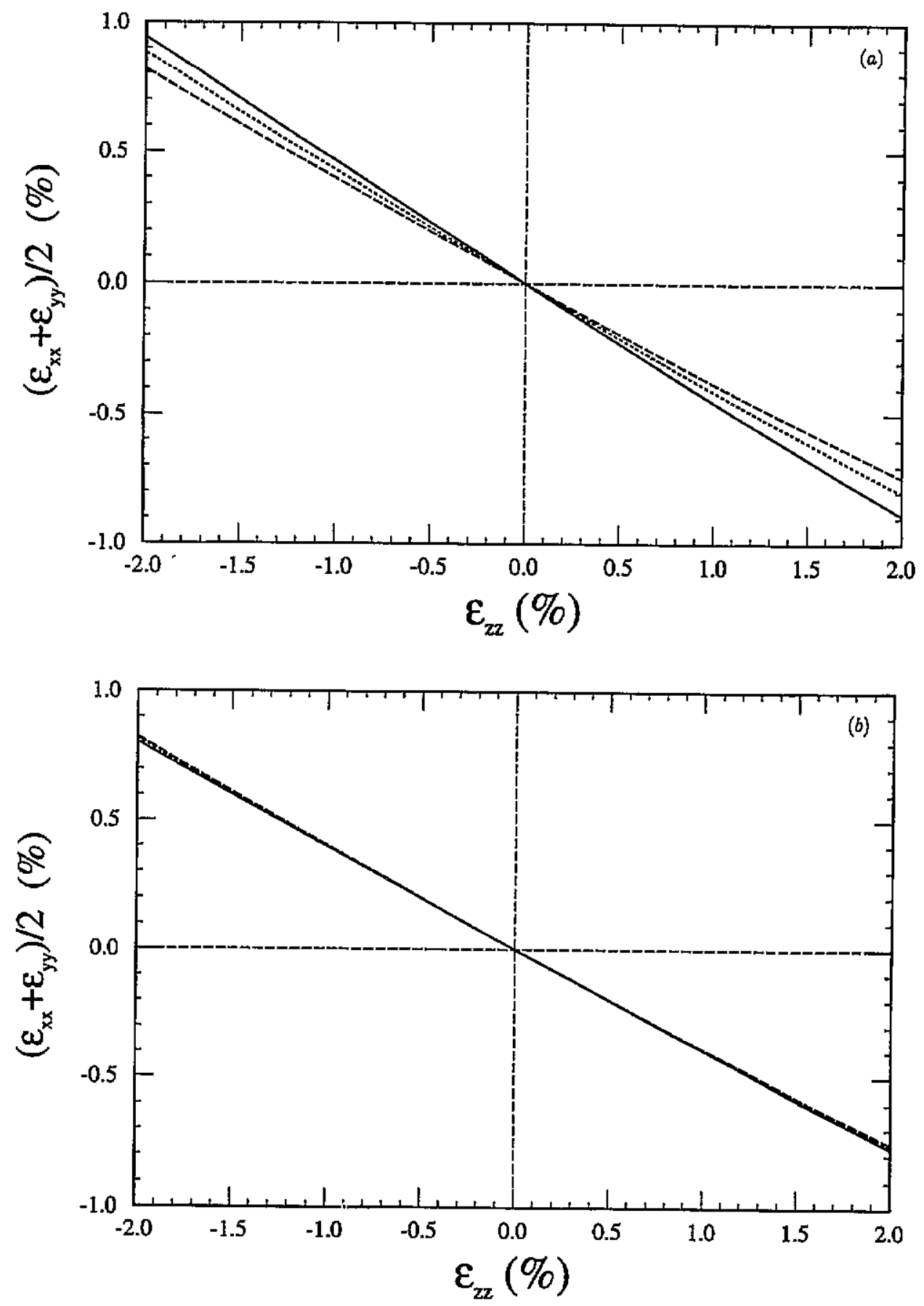

Figure 4. The variation in the transverse strain $\left(\varepsilon_{x x}+\varepsilon_{y y}\right) / 2$ as a function of the normal strain $\varepsilon_{z z}$ as determined for the loading directions in figure 3 employing the (a) Au potential and (b) the 'elastically isotropic' Au 1 potential,' 
versus normal strain curves (negative Poisson ratio) in figure $4(b)$ are the same, while for an anisotropic crystal the slopes are different as seen in figure $4(a)$. These results taken in toto demonstrate that the modified interatomic potentials do produce elastically isotropic crystals.

\section{Surface properties}

In order to determine the effects of the modifications of the Ag and Au interatomic potentials on the properties of defects, we have performed a series of calculations ( $T=0$ energy minimizations) on the (001), (011) and (111) surfaces of Ag and Au using the 'elastically anisotropic' and 'elastically isotropic' interatomic potentials. We note that the modifications of the interatomic potentials necessary to describe an isotropic solid affects both the elastic constants and the anharmonicity of the interatomic potential. Since different surfaces have different relaxation symmetries, they should be controlled by different combinations of these two effects.

To determine the surface properties, we employed a slab geometry for the simulation cell and used periodic boundary conditions in the plane parallel to the slab surface. For all surfaces studied here, the slab thickness was more than 50 atomic planes. We equilibrate the surfaces by minimizing the total energy of the slab with respect to the positions of all of the atoms employing a conjugate gradient method. The relaxed and unrelaxed surface energies for the Au and two pseudo-Au potentials and those corresponding to Ag are listed in table 3. The Au and Ag surface energies (second column) are slightly different (within approximately $10 \%$ ) from those recently reported for this potential [5] due to small changes in the knot points used in the present study and the manner in which the coefficient $a_{6}$ was determined.

Table 3. Surface energies determined employing the potentials in table 2 . The values in parentheses are the unrelaxed surface energies.

\begin{tabular}{llll}
\hline & \multicolumn{3}{c}{ Surface energy $\left(\mathrm{mI} \mathrm{m} \mathrm{m}^{-2}\right)$} \\
\cline { 2 - 4 } & Anisotropic & Isotropic I & Isotropic II \\
\hline $\mathrm{Au}(001)$ & $744.338(783.109)$ & $728.183(768.239)$ & $732.189(766.975)$ \\
$\mathrm{Au}(011)$ & $794.357(880.479)$ & $800.705(881.088)$ & $807.235(879.821)$ \\
$\mathrm{Au}(111)$ & $647.738(684.692)$ & $678.443(710.917)$ & $680.402(709.962)$ \\
& & & \\
$\mathrm{Ag}(001)$ & $752.052(760.007)$ & $740.056(749.403)$ & $744.492(748.077)$ \\
$\mathrm{Ag}(011)$ & $814.941(844.633)$ & $825.152(852.355)$ & $835.757(851.131)$ \\
$\mathrm{Ag}(111)$ & $657.887(672.292)$ & $696.643(708.845)$ & $700.194(708.128)$ \\
\hline
\end{tabular}

The (001) surface energies determined for the $\mathrm{Au}$ and $\mathrm{Ag}$ anisotropic potentials are lower than those obtained for their corresponding isotropic potentials while the reverse trend is observed for the (011) and (111) surfaces (compare the second column with the third and fourth columns in table 3 ). The same trends also hold for the unrelaxed surface energies (see table 3). If we take the ratio of the (001) to (111) surface energies (or the (011) to (111) surface energies) as a measure of the surface energy anisotropy $\Gamma$ the results presented here show that the $\Gamma$-values for the 'elastically isotropic' potentials $(\Gamma \approx 1.07$ and 1.06 for $\mathrm{Au}$ and $\mathrm{Ag}$, respectively) are closer to unity than those corresponding to the elastically anisotropic potentials ( $\Gamma \approx 1.15$ and 1.14 for $\mathrm{Au}$ and $\mathrm{Ag}$, xespectively). Therefore, the results determined for these potentials suggest that there is a correlation between elastic and 
surface energy anisotropies; however, the present data are insufficient to prove that this is a general trend.

Table 4. Changes in the spacing between the five atomic planes closest to the surface determined employing the potentials given in table 2 . $\Delta d_{i}$ is the change in the spacing between the $i$ th and $(i+1)$ th planes upon surface relaxation ( $i=1$ denotes the surface plane), A, I and II denote elastically 'anisotropic' and 'isotropic' I and II potentials, respectively.

\begin{tabular}{|c|c|c|c|c|c|c|c|c|c|}
\hline & \multicolumn{9}{|c|}{ Change $(\%)$ in spacing } \\
\hline & \multicolumn{3}{|c|}{$(001)$} & \multicolumn{3}{|c|}{$(011)$} & \multicolumn{3}{|c|}{ (111) } \\
\hline & $A$ & I & II & A & I & II & $A$ & I & II \\
\hline$A u \Delta d_{l}$ & -5.33 & -5.09 & -5.01 & -13.12 & -12.13 & -12.01 & -4.30 & -3.76 & -3.74 \\
\hline Au $\Delta d_{2}$ & 1.77 & 1.92 & 1.77 & 2.44 & 3.00 & 2.71 & 1.39 & 1.05 & 0.97 \\
\hline Au $\Delta d_{3}$ & -0.54 & -0.71 & -0.62 & 1.11 & -0.01 & 0.09 & -0.40 & -0.26 & -0.23 \\
\hline Au $\Delta d_{4}$ & 0.17 & 0.26 & 0.22 & -0.85 & -0.23 & -0.24 & 0.12 & 0.07 & 0.06 \\
\hline Ag $\Delta d_{1}$ & -3.01 & -3.04 & -2.02 & -8.75 & -8.01 & -6.19 & -3.20 & -2.69 & -2.30 \\
\hline $\operatorname{Ag} \Delta d_{2}$ & 0.50 & 0.78 & 0.40 & -0.03 & 0.88 & 0.08 & 0.67 & 0.47 & 0.33 \\
\hline $\mathrm{Ag} \Delta d_{3}$ & -0.08 & -0.21 & -0.10 & 1.16 & 0.27 & 0.41 & -0.13 & -0.08 & -0.04 \\
\hline Ag $\Delta d_{4}$ & 0.01 & 0.06 & 0.02 & -0.50 & -0.17 & -0.19 & 0.02 & 0.01 & 0.01 \\
\hline
\end{tabular}

Surface relaxation changes the spacings between consecutive atomic planes parallel to the surface from the spacing $d_{0}$ between planes in the perfect crystal. The changes in the interplanar spacings $\Delta d_{i}$ (i.e. the spacing between the $i$ th and $(i+1)$ th planes from the surface minus $d_{0}$, where $i=1$ corresponds to the surface plane), for the five atomic planes closest to the surface are listed in table 4. For all six potentials, the $\Delta d_{i}$-values for the (001) and (111) surfaces show the same qualitative behaviour. There is a contraction between the top two atomic planes followed by an expansion between the next two planes. This alternation in the sign of the relaxation continues with a decaying amplitude for the next several planes. The $\Delta d_{i}$-values for the (011) surfaces show the same type of oscillatory behaviour as for the other surfaces; however, the wavelength of the oscillation is greater than twice the interplanar spacing. The surface relaxation (figure 5) obtained with the "elastically anisotropic' potentials is somewhat larger and extends further into the bulk than is found when the 'elastically isotropic' potentials are employed.

The fact that the surface relaxations obtained using the 'elastically isotropic' and 'elastically anisotropic' potentials are very similar in the high-symmetry (001) and (111) surface suggests that surface relaxation, in these cases, is dominated by the differences between the anharmonicities of the potentials. On the other hand, the less good agreement between the surface relaxation results obtained with the 'anisotropic' and 'isotropic' potentials on the (011) surface suggests that in lower-symmetry situations the differences may be attributable largely to the differences in elastic constants.

\section{Summary}

We have developed interatomic potentials to represent elastically isotropic solid models. The potentials are of the FS type and are parametrized to fit Au and Ag. Two different choices for making the potentials isotropic are examined. Under the uniaxial load condition, it was shown that the solids modelled employing the "elastically isotropic' potentials remain elastically isotropic for strains of $0.5 \%$ or less. The properties of the $(001),(011)$ and 


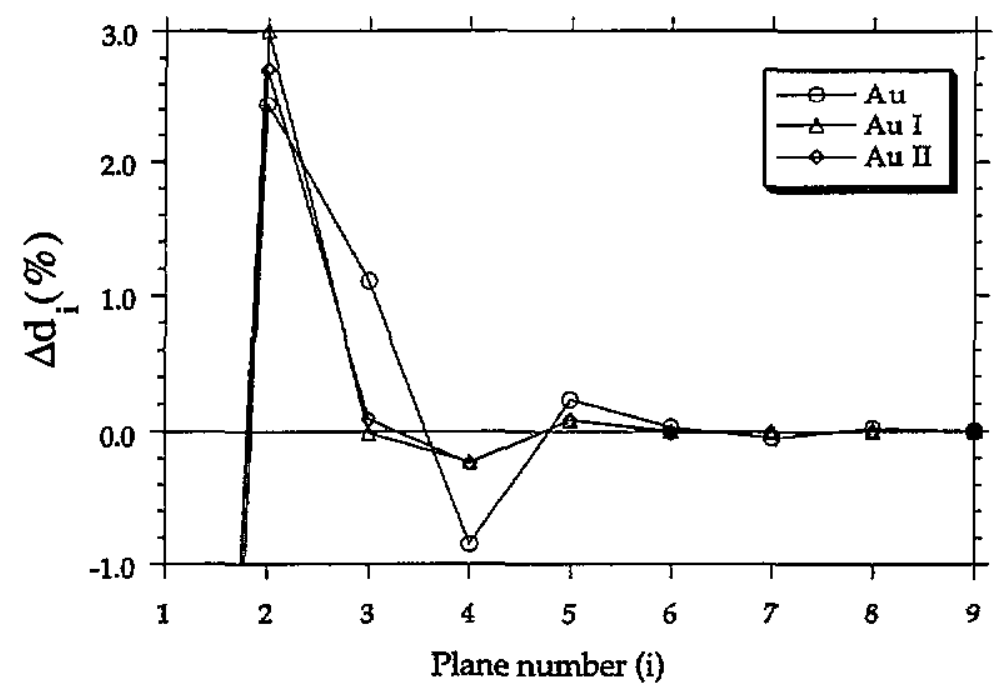

Figure 5. Changes in the (011) interplanar spacing $\Delta d_{1}$ as a function of plane number $(i=1$ denotes surface plane).

(111) surfaces were determined and compared with the those of elastically anisotropic $\mathrm{Au}$ and $\mathrm{Ag}$. It was shown that the surface energies and structures determined employing the 'elastically anisotropic' potentials are similar to those obtained for their corresponding 'elastically isotropic' potentials. It was also found that the surface energy anisotropy is lower in solids modelled employing 'elastically isotropic' potentials than in those represented by elastically anisotropic potentials.

\section{Acknowledgments}

The authors gratefully acknowledge the support of the Division of Materials Science of the Office of Basic Energy Sciences of the US Department of Energy under grant FG0288ER45367.

\section{References}

[1] Sinclair J E, Gehlen P C, Hoagland R G and Hirth J P 1978 J. Appl. Phys. 493890

[2] Najafabadi R and Srolovitz D J 1995 unpublished research

[3] Finnis M W and Sinclair J E 1984 Phil Mag. A 5045

[4] Daw M S and Baskes M I 1984 Phys. Rev. B 296443

[5] Ackland G J, Tichy G, Vitek V and Finnis M W 1987 Phil. Mag. A 56735

[6] Rose J H, Ferrante J and Smith J R 1981 Phys. Rev. Lett. 47675 